See discussions, stats, and author profiles for this publication at: https://www.researchgate.net/publication/282890942

\title{
Viruses as Model Nanoreactors to Study Enzyme Kinetics
}

Chapter · September 2015

DOI: 10.1201/b18970-4

3 authors, including:

W. Frederik Rurup

University of Twente

9 PUBLICATIONS 148 CITATIONS

SEE PROFILE 


\title{
Viruses as model nanoreactors to study enzyme kinetics
}

\author{
W. Rik Rurup, Melissa S. Koay, Jeroen J. L. M. Cornelissen \\ Department of Biomolecular Nanotechnology \\ MESA+ Institute for Nanotechnology \\ University of Twente, Enschede, The Netherlands \\ j.j.l.m.cornelissen@utwente.nl
}

In nature, the interior of a cell hosts a remarkable network of organelles and sub-cellular compartments in which many biochemical reactions take place. From membrane-based assemblies (such as the mitochondrion, Golgi apparatus, vesicles and peroxisomes) to non-membrane enclosed ribosomes the compartmentalization of enzymes, substrates and cofactors within a confined environment allows the cell to gain excellent spatial and temporal control over biochemical pathways.[1-3] This organization leads to a significant enhancement in substrate turnover and catalytic efficiency. As a consequence, there is much interest in the field of nanotechnology to develop enzyme nanoreactors that mimic the natural organization of cells and organelles. However this involves two key challenges: 1) the precise arrangement of enzymes in space and time, and 2) mimicking the molecular crowding effects imposed by the confined environment. While recent advances using top-down nanolithography approaches provide significant spatial and temporal control, it is increasingly evident that the confined environment plays a crucial role in optimising the catalytic efficiency.[4]

Book Title

First Author \& Second Author

Copyright (C) 2009 by Pan Stanford Publishing Pte Ltd

www.panstanford.com 
For these reasons, much effort focused towards the development of enzyme nanoreactors, in which the enzymes are either tethered to the exterior of a nanosized assembly, or encapsulated inside. While there are several examples of enzyme nanoreactors based on biosynthetic assemblies such as nanoparticles, nanopores, polymersomes and vesicles, $[5,6]$ such assemblies are often highly concentration-dependent, display limited substrate selectivity and are difficult to precisely control the positioning of enzymes.[7] In recent years, much attention has turned towards protein-based assemblies, particularly viruses, as an alternative building block for the assembly of model nanoreactors.[7-12]

\section{GLOSSARY}

Capsid: a protein shell that encapsulates the genetic material (RNA/DNA) of a virus. The virus capsid is formed by the selfassembly of multiple copies of capsid coat protein monomers into large supramolecular structures (typically rod-shaped assemblies or icosahedral bodies).

Coiled-coil: a common structural motif in protein-protein interactions. Coiled-coils are formed by tandem heptad sequence repeats that form $\alpha$-helices. Two or more $\alpha$-helices interact to form supercoiled structures. The heptad sequence repeats, namely abcdefg, display hydrophobic residues at positions $a$ and $d$, whereas $e$ and $g$ are frequently occupied by charged residues that direct the orientation of the $\alpha$-helices in the coiled-coil.

DNA aptamer: short DNA stranded oligonucleotides that specifically bind a target molecule.

Virus-based enzyme nanoreactor: virus protein-cage assemblies that are designed to carry enzymes either inside or on their surface.

Virus-like particles (VLPs): are produced by the self-assembly of capsid protein monomers into large supramolecular assemblies. Such assemblies are morphologically similar to the capsid, however, since it is devoid of the virus genetic material, such assemblies are noninfectious. 


\subsection{INTRODUCTION}

Viruses are intrinsically attractive scaffolds due to their ability to self-assemble into highly stable symmetrical assemblies.[13] Composed of multiple copies of protein monomers, virus capsids can be readily modified (either chemically or genetically) and functionalized, making them highly versatile and tuneable scaffolds. While there are many examples of viruses as nanocarriers and (targeted) drug delivery systems, their potential applications as enzyme nanoreactors has only recently emerged. This chapter provides an overview of the different virus building blocks used in the development of enzyme nanoreactors, and deals with the challenges one faces when studying enzymes within a confined environment,[14] in particular how the classical laws of enzyme kinetics may no longer apply under such conditions and how viruses could be used as nanoreactors to model biological organelles.

\subsection{VIRUS-BASED BUILDING BLOCKS FOR NANOREACTORS}

Although viruses can be categorized into different morphological classes, the most commonly virus assemblies used in nanotechnology are those of icosahedral or rod-shaped viruses (Figure 5.1). In particular, the plant-based icosahedral Cowpea Mosaic Virus (CPMV) and Cowpea Chlorotic Mottle Virus (CCMV) and the rod-shaped Tobacco Mosaic Virus (TMV) are amongst the most widely used and most well characterized viruses in nanotechnology.[1, 15-27]

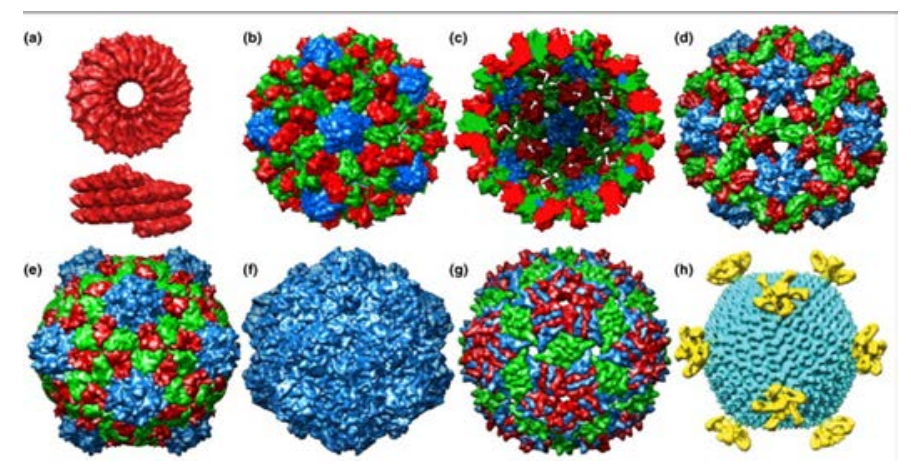

Figure 5.1. Some of the viruses currently used as model enzyme 


\subsubsection{Plant-based viruses}

For the use of viruses as enzyme nanoreactors, the porosity of the capsid shell plays an important role in the diffusion of substrates and products. In particular, one of the most attractive features $\mathrm{w}$ a stable virus assembly that contains pores that can be reversibly controlled or triggered (for example, by altering the $\mathrm{pH}$ and ionic strength). By tuning and controlling the $\mathrm{pH}$, the gradual increase in shell porosity could be extremely useful for the selective uptake and release of substrates and products. For example, Cowpea Chlorotic Mottle Virus (CCMV) is composed of 180 identical copies of capsid coat protein that self-assemble at $\mathrm{pH} 5.0$ to form the intact $\mathrm{T}=3$ capsid with an external diameter of $28 \mathrm{~nm}$. At $\mathrm{pH}$ 5.0, CCMV capsids contain small $<1 \mathrm{~nm}$ pores on the threefold axis and $<0.5 \mathrm{~nm}$ on the fivefold axis, respectively (Figure 5.2). At near physiological ( $\mathrm{pH} \sim 7.0)$, a $10 \%$ swelling occurs around the pores, while the virus assembly itself remains stable. During this process, the porosity increases to $\sim 2 \mathrm{~nm}$ on the quasi-threefold axis, that allows diffusion of larger molecules.[25] At $\mathrm{pH}>7.5$, the CCMV capsid disassembles completely yielding free capsid coat proteins. While the $\mathrm{pH}$ driven swelling is an attractive approach to reversibly trigger changes in the porosity, gaining precise control over the pore size is extremely difficult.

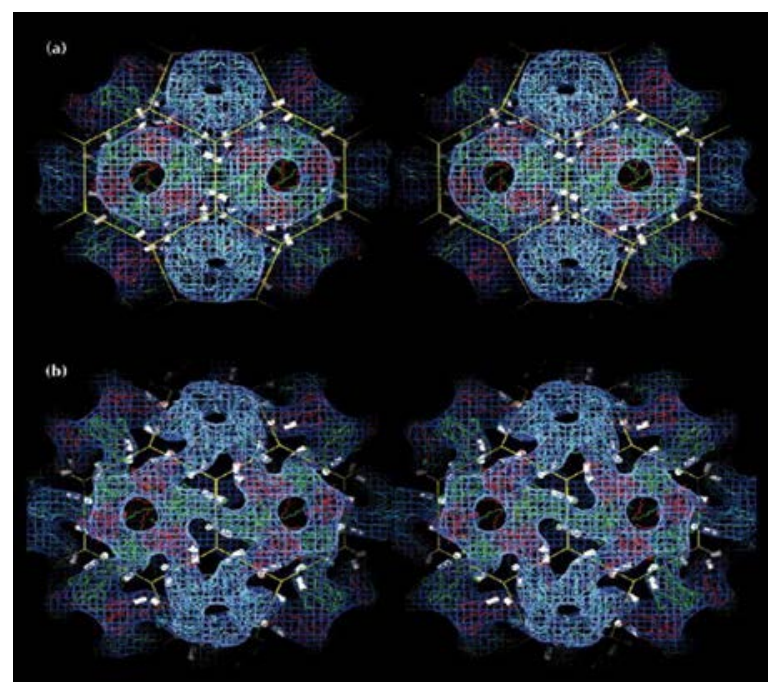

Figure 5.2. Stereoviews of Cowpea Chlorotic Mottle Virus show the capsid swelling upon increasing the $\mathrm{pH}$ from (a) $\mathrm{pH} 4.5$ to (b) $\mathrm{pH} 7.5$, resulting in an increase in capsid pore size. 


\subsubsection{Bacteriophages}

In recent years, MS2, Q $\beta$ and P22 bacteriophages have become increasingly used as an alternative scaffold for virus-based assemblies. Offering intrinsically unique properties, these bacteriophages are more stable than plant-based viruses, yet can also form empty capsid shells, making them an attractive series of virus-like nanoreactors (Figure 1). The native MS2 virion is composed of three components: the virus coat protein $(13.7 \mathrm{kDa})$, the A protein $(44.0 \mathrm{kDa})$ and a single-stranded RNA molecule. Similar to the plant-based viruses, MS2 can be reassembled from acid-driven disassembled monomers into a stable virus-like particle. Moreover, the MS2 shell possesses 32 pores per capsid, each approximately $1.8 \mathrm{~nm}$ in diameter, which allows the inclusion of potential substrates and catalysts.[26] Interestingly Tullman-Ercek and coworkers showed that by using osmolytes such as trimethyl amine $\mathrm{N}$-oxide (TMAO), the encapsulation efficiency of enzymes was enhanced.[28] Although osmolytes are used to regulate osmotic pressure inside cells, they have also been shown to be highly effective in stabilizing proteins.[29, 30] The use of osmolytes is an interesting approach to alter the capsid porosity, which could be used to confer substrate selectivity or regulate the diffusion of inhibitory molecules.

P22 is a rather unique bacteriophage that is related to bacteriophage $\lambda$. Composed of 420 coat proteins, P22 recruits 100-330 scaffold proteins in order to assemble into the icosahedral $\mathrm{T}=7$ procapsid of $58 \mathrm{~nm}$ diameter. Upon heating to $65^{\circ} \mathrm{C}$ for 10 minutes, the P22 procapsid (PC) transforms to the mature capsid, whereby the capsid swells to $64 \mathrm{~nm}$ and the scaffold proteins are released. Interestingly, although the interior volume of the capsid is doubled in this expanded form (EX), the pore size of $2 \mathrm{~nm}$ remains unaffected. Upon raising the temperature further $\left(75^{\circ} \mathrm{C}\right.$ for 20 minutes), 12 pentamers are released, irreversibly creating a wiffle ball (WB) capsid with large $10 \mathrm{~nm}$ sized holes (Figure 5.3). In this form, free diffusion of substrates in and out of the capsid is reported.[7]
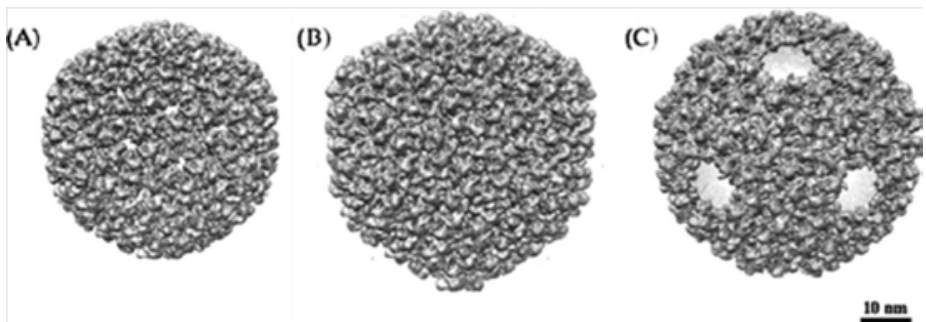

Figure 5.3. Swelling of bacteriophage P22 leads to different capsid forms 


\subsection{ENZYME LOADING STRATEGIES}

While virus-like particles form well-defined assemblies, the controlled loading of enzymed is a significant challenge that cannot be easily overcome. In the past, statistical encapsulation was used to encapsulate horseradish peroxidase inside CCMV.[25] While convenient, statistical encapsulation is a highly concentration dependent process and hence is largely uncontrollable and often irreproducible. Since the internal walls of many viruses are highly positively-charged, negatively-charged linkers have been since been explored to promote charge-charge interactions for the controlled in vitro and in vivo loading of enzyme cargo inside virus assemblies:

\subsubsection{In vitro loading}

\section{Coiled coil peptides}

In CCMV, heterodimerizing 'coiled-coil' peptides were designed by fusing a positively-charged $\alpha$-helix to the $\mathrm{N}$-terminus of the CCMV capsid coat protein (Figure 5.4a). Fusion of a complementary negatively-charged $\alpha$-helix to the $C$-terminus of lipase $B$ from Pseudozyma antartica promoted the formation of a non-covalent lipase BCCMV complex. By varying the mixing ratios of enzyme capsid complex with wild type capsid protein the amount of loaded enzyme could be influenced.[31] Examples: CCMV, CPMV.

\section{Charge induced}

Inspired by the natural loading of DNA/RNA cargo, the use of nucleic acid based linkers and their mimics have become increasingly popular to gain controlled loading. For example, MS2 bacteriophages rely on a targeting RNA-hairpin for the internalization of its genomic RNA. Tullman-Ercek and coworkers recently mimicked this approach to target a non-native enzyme inside MS2 capsids.[28] In this case, a poly (16) anionic peptide linker was used as a RNA mimic, which was fused to the C-terminus of E. coli alkaline phosphatase (PhoA) to facilitate loading inside MS2 (Figure 5.4b). Examples: bacteriophage MS2 
(a) Coiled coil

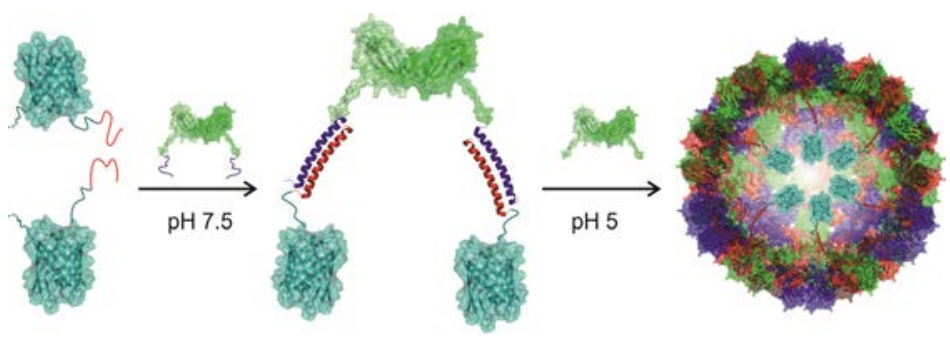

(b) Charge induced

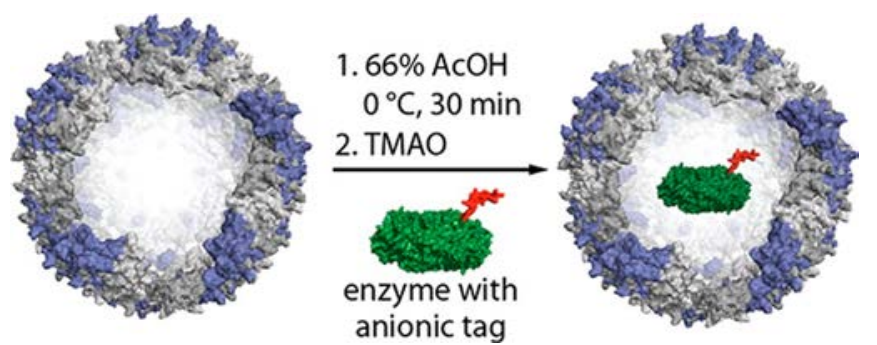

(c) DNA aptamer

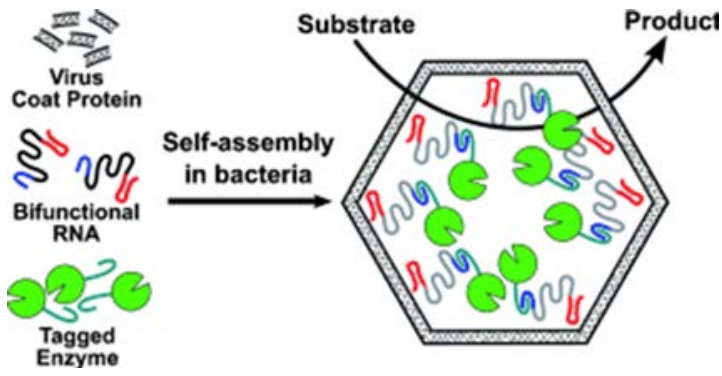

(d) Scaffold protein

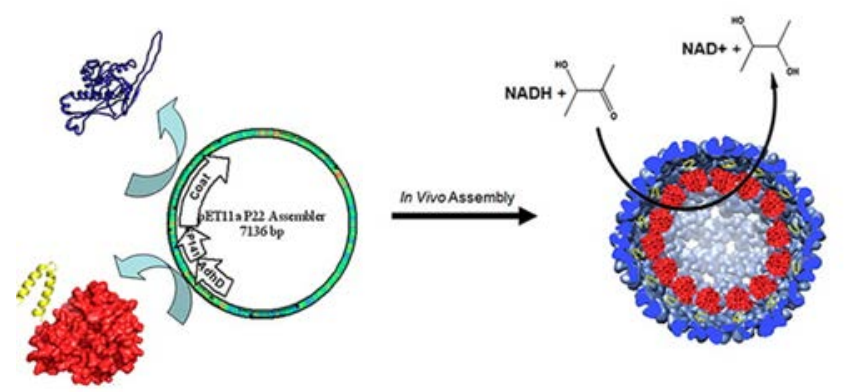

Figure 5.4. In vitro and in vivo enzyme loading strategies. 


\subsubsection{In vivo loading}

\section{DNA aptamers}

In some cases, the use of specific RNA/DNA recognition loops allows further control and selectivity over cargo loading. An elegant example is that of the recombinant $Q \beta$ bacteriophage, which could be loaded and assembled recombinantly under in vivo conditions. This design approach comprised of two plasmids of which the first encodes expression of the virus capsid, the second the target enzyme. The mRNA used to transcribe the capsid protein was subsequently used as an anchor. The native RNA docking sequence was engineered on the $3^{\prime}$-primer end and on the $5^{\prime}$-end, an $\langle-R e v$ aptamer was used. By fusing the N-terminal Revtag to the target enzyme, the two primer ends form "baits" for Q $\beta$ ( $3^{\prime}$-end) and the target enzyme (5'-end). This DNA-aptamer system has already been successfully used to encapsulate peptidase E, luciferase and a thermostable luciferase variant,[8] making it a versatile and effective approach for the in vivo loading of enzymes inside virus-based assemblies (Figure 5.4c). Furthermore, by tuning the recombinant expression conditions, the amount of encapsulated enzyme can be influenced, which allows further control over enzyme loading inside bacteriophage Q $\beta$. Examples: bacteriophages MS2, Q $\beta$

\section{Signaling proteins}

Of the viruses discussed thus far, P22 and SV40 are the only examples that rely on a scaffold protein during assembly (Figure 5.4d). For P22, a truncated version of the scaffold protein fused to a target protein was shown to be sufficient to encapsulate enzyme cargo during in vivo assembly. After forming the extended (EX) form, the scaffold proteins protrude out of the virus assembly which can be subsequently removed by enzymatic digestion. Similarly, the capsid of the SV40 major capsid protein has a strong interaction with amino acids 273-308 of minor capsid protein 2. Co-infection of baculoviruses expressing either the SV40 coat protein or the cargo protein results in stable, enzyme loaded capsids in vivo. By using their respective signaling proteins, alcohol dehydrogenase D (AdhD) from Pyrococcus furiosus, the homo-tetrameric CelB and yeast cytosine deaminase (yCD) have all been successfully 
encapsulated in P22 and SV40, respectively.[7, 32] However, the controlled enzyme loading have not been reported for either system.

Examples: bacteriophage P22, SV40

\section{Essential differences in loading strategies}

Most encapsulation strategies are inspired based on native virus loading mechanisms. To date, the different loading strategies can be classified in two broad groups: in vitro or in vivo loading. For viruses with a known assembly/disassembly pathway, in vitro loading mechanisms offer a versatile approach for the controlled encapsulation of enzymes. The main benefit of in vitro loading is the possibility to purify the separate components before encapsulation. A secondary benefit is the enzyme loading can be more tightly controlled (and in some cases, predictive loading could also be possible). In contrast, for viruses and virus-like assemblies that undergo in vivo loading are advantageous since many of these assemblies are intrinsically more stable. However, one major disadvantage is that there is little control over the number of enzymes encapsulated, which is likely to lead to a disperse distribution of enzyme loading between virus capsids.

\subsection{ENCAPSULATION EFFECTS}

In nature, the cell membrane provides a physical boundary to confine enzymes and substrates within a local environment. This confinement imposes a large molecular crowding effect, whereby the reduced volume increases the effective concentration of enzymes and significantly improves the kinetic efficiency of enzymes.[27, 33, 34] Similarly, the virus capsid provides a confined environment, however, the effects of encapsulating enzymes inside virus nanoreactors thus far, is very diverse.

\subsubsection{CCMV}

The encapsulation of Pseudozyma antartica lipase B (PalB) inside CCMV was shown to increase the initial velocity with respect to the free enzyme in bulk solution, which may suggest that the efficiency or turnover rate of substrate is enhanced upon encapsulation. However interestingly, the same study also showed that encapsulation of more than one enzyme 
within the same capsid decreased the reaction rate, suggesting that, for CCMV, molecular crowding plays a significant and almost immediate role on the encapsulated enzyme. In another example, horseradish peroxidase (HRP) was encapsulated inside CCMV. However, while no kinetic analyses were performed, it was shown that increasing the $\mathrm{pH}$ to induce capsid swelling also led to increased product diffusion.[35]

\subsubsection{Bacteriophages $M S 2, Q \beta$}

Although the MS2 capsid is similar in size to CCMV (27 nm), it is unique as it contains 32 pores ( $1.8 \mathrm{~nm}$ in diameter), that allows diffusion of large substrates into the capsid without disassembling the virus (unlike CCMV). [36] The encapsulation of (on average) 1-2 alkaline phosphatase (PhoA) inside the MS2 bacteriophage resulted in only a marginal increase in $K_{m}$ and a decrease in $\mathrm{k}_{\text {cat. }}$ [28] Due to the size of PhoA $(94.9 \mathrm{kDa})$, Glasgow et al. attributed this decrease in enzyme efficiency to spatial crowding inside the MS2 capsid, and is a striking example of the delicate interplay between enhancement and inhibition of enzymes in confined environments.

In another example, Fiedler et al. reported the encapsulation of aspartate dipeptidase peptidase E (PepE) in bacteriophage Q $\beta$.[8] In this example, the catalytic efficiency $\left(k_{c a t} / \mathrm{K}_{\mathrm{m}}\right)$ for the encapsulated PepE (2-18 enzymes per virus-like particle) increased 3-fold compared to free PepE. Importantly, this work showed that encapsulated PepE exhibited significantly improved thermostabilization. After heating to $50^{\circ} \mathrm{C}$ for 30 minutes, no loss in activity was observed for the encapsulated PepE, whereas under the same conditions, free PepE retained only 20\% activity. Similarly, encapsulation was also shown to effectively protect PepE from enzymatic digestion by proteinase K (retaining $80 \%$ activity). In the same work, luciferase (Luc) and a thermostable luciferase (tsLuc) encapsulated in $Q \beta$ displayed an increase in $K_{m}$ (i.e. increased apparent substrate binding affinity), suggesting that indeed the effective local substrate concentration increases inside the capsid. Interestingly however, no enhancement of $k_{\text {cat }}$ was observed. The authors suggested that perhaps the enzyme flexibility (or a restriction thereof) might cause this negative influence on kinetic performance. 


\subsubsection{Bacteriophage P22}

The dense packing of approximately 250 alcohol dehydrogenase D (AdhD) enzymes per P22 bacteriophage led to an effective confinement molarity ( $\left.\mathrm{M}_{\text {conf }}\right)$ of $7.16 \mathrm{mM}$.[7] Significantly, Douglas and coworkers reported that $250 \mathrm{AdhD}$ led to $\sim 27 \%$ occupancy of the total inner volume of the P22 capsid. This is a remarkable example since it closely resembles the conditions of the cell, whereby the overall concentration of all enzymes and macromolecules combined is also estimated to occupy up to $30 \%$ of the physically available space.[27, 33, 37, 38] Intriguingly, the apparent $\mathrm{k}_{\text {cat }}$ was reported to be lower compared to the free enzyme and the overall enzyme efficiency $\left(k_{\text {cat }} / \mathrm{K}_{\mathrm{m}}\right)$ also decreased. Similar observations were reported for the encapsulation of the homotetrameric $\beta$-glycosidase (CelB).[11] Encapsulation of 84-87 CelB monomers (21 homotetramers) per P22 showed no significant change in efficiency $\left(k_{\text {cat }} / \mathrm{K}_{\mathrm{m}}\right)$.

Table 1.1. Overview of the confined kinetic effects observed in some model nanoreactors

\begin{tabular}{|c|c|c|c|c|c|}
\hline VIRUS & $\begin{array}{l}\text { PORE } \\
\text { SIZE }\end{array}$ & $\begin{array}{l}\text { ENZYME } \\
\text { (E) }\end{array}$ & $\begin{array}{l}\text { SUBSTRATE } \\
\text { (S) }\end{array}$ & $\# \mathrm{E}$ & KINETIC EFFECTS \\
\hline \multirow[t]{4}{*}{ CCMV } & $<1 \mathrm{~nm}$ & PalB & DiFMU & 1.3 & 5 fold increase \\
\hline & $<1 \mathrm{~nm}$ & PalB & DiFMU & 4.0 & No change \\
\hline & $<1 \mathrm{~nm}$ & HRP & Rhodamine & 1.0 & 2-3 fold increase \\
\hline & $2 \mathrm{~nm}$ & HRP & Rhodamine & 1.0 & No change \\
\hline MS2 & $1.8 \mathrm{~nm}$ & PhoA & PNPP & 1.6 & Slight decrease \\
\hline \multirow[t]{4}{*}{$\mathrm{Q} \beta$} & $1.4 \mathrm{~nm}$ & PepE & Asp-AMC & 9.0 & No change \\
\hline & & Luc & Luciferin & 4.0 & Lower $\mathrm{k}_{\text {cat }}$ higher $\mathrm{K}_{\mathrm{m}}$ \\
\hline & & tsLuc & Luciferin & 2.0 & Equal $\mathrm{k}_{\mathrm{cat}}$, higher $\mathrm{K}_{\mathrm{m}}$ \\
\hline & & tsLuc & Luciferin & 9.0 & Lower $\mathrm{k}_{\text {cat, }}$,higher $\mathrm{K}_{\mathrm{m}}$ \\
\hline \multirow[t]{2}{*}{ P22 (PC) } & $2 \mathrm{~nm}$ & AdhD & Acetoin & 250 & Lower $\mathrm{k}_{\text {cat, }}$ lower $\mathrm{K}_{\mathrm{m}}$ \\
\hline & $2 \mathrm{~nm}$ & CelB & PNPG & 85 & No change \\
\hline \multirow[t]{2}{*}{ P22 (EX) } & $2 \mathrm{~nm}$ & AdhD & Acetoin & 250 & $\mathrm{k}_{\text {cat, }}$ same, higher $\mathrm{K}_{\mathrm{m}}$ \\
\hline & $2 \mathrm{~nm}$ & CelB & PNPG & 85 & No change \\
\hline \multirow[t]{2}{*}{ P22 (WB) } & $10 \mathrm{~nm}$ & AdhD & Acetoin & 250 & No change \\
\hline & $10 \mathrm{~nm}$ & CelB & PNPG & 85 & No change \\
\hline
\end{tabular}

In general, there appears to be a trend between the virus size and the enzyme loading number with its overall efficiency as an enzyme 
nanoreactor. Smaller viruses such as CCMV, Q $\beta$ and MS2, which are in the order of 24-28 nm in diameter, experience enhanced efficiency when less enzyme is encapsulated. Minten et al showed that encapsulation of more than one PalB in the same CCMV capsid, decreased the overall reaction rate. This suggests that molecular crowding may play a significant and almost immediate role on the encapsulated enzymes in such virus assemblies.

In contrast, the number of enzymes encapsulated in larger virus assemblies such as P22 is typically high (85-250 enzymes per capsid). However, despite the absolute number of encapsulated enzymes, these conditions closely resemble true molecular crowding and volume occupancy found in cells. Surprisingly, under these conditions, the overall efficiency and the apparent affinity were both reported to be lower compared to the free enzyme. Nevertheless, it should be noted that, unlike the cell, whereby the actual concentration of a single enzyme in a cell is typically low, the encapsulation of a high number of the same enzyme is also likely to lead to self-inhibition.

\subsection{SUBSTRATE DIFFUSION}

The virus capsid not only provides a confined environment but also creates a physical barrier, which may influence the diffusion of substrates across the capsid shell and may differ compared to traditional membrane-based organelles. There are currently few examples reporting the substrate diffusion rates between different viruses. Comellas-Aragones et al showed that the diffusion of rhodamine 6G in and out of CCMV is hindered for smaller pore sizes, whereas for the larger pore sizes $(2 \mathrm{~nm})$ free diffusion was observed.[25] Recently, Douglas and coworkers reported the same observation for the bacteriophage P22.[7] In this work, it was shown that increasing the pore size from $2 \mathrm{~nm}$ to $10 \mathrm{~nm}$ did not result in an increase in substrate and cofactor diffusion, suggesting that a pore size of $2 \mathrm{~nm}$ is sufficient to allow free diffusion across the capsid.

\subsection{FUTURE OUTLOOK}

It has been shown that substrate diffusion, enzyme folding (and hence apparent substrate binding affinity, $\mathrm{K}_{\mathrm{m}}$ ) and enzyme immobilization 
play important roles in the overall enzyme efficiency $\left(\mathrm{K}_{\text {on }} / \mathrm{K}_{\text {off }}\right.$ rates). Similarly, the current approaches to encapsulate enzymes inside viruses typically involve chemical or genetic modification or tethering of the enzyme which may not only restrict the enzyme activity but also the rotation and conformation of the enzyme itself. Currently, all models have thus far assumed normal Michaelis-Menten enzyme kinetics. However, it is becoming increasingly apparent that standard Michaelis-Menten kinetics and hence, the standard assumptions may not be valid for enzymes that are within a confined environment. In particular, two parameters, namely the "free-diffusion" assumption and the apparent affinity $\left(\mathrm{K}_{\mathrm{on}} / \mathrm{K}_{\text {off }}\right.$ rates) need to be re-evaluated for confined systems. While fractal kinetic theory has been used to describe enzyme kinetics within the confined environment of cells and organelles, the exact parameters that define such models and its relevance to biochemical pathways are still currently under debate. In the meantime, alternative enzyme loading strategies that do not alter or modify the enzyme itself, or reversible linkers (i.e. photocleavable linkers) could be further explored.

Nevertheless, in recent years, more virus-like assemblies such as encapsulins, carboxysomes and de novo nano-containers (such as lumazine synthase from Aquifex aeolicus) have emerged as highly promising examples for the future generation of enzyme nanoreactors. Nonetheless, the examples that currently exist for virus nanoreactors have already contributed greatly towards understanding the confined environment of organelles and cells. The enhanced efficiency of enzymes, combined with the protective properties seen in the different systems described above, still only hint at the possibilities for future enzyme nanoreactors.

\subsection{REFERENCES}

[1] Cardinale, D., Carette, N. and Michon, T. Virus scaffolds as enzyme nano-carriers. Trends Biotechnol., 30, 7 (Jul 2012), 369-376.

[2] Tanaka, S., Sawaya, M. R. and Yeates, T. O. Structure and Mechanisms of a Protein-Based Organelle in Escherichia coli. Science, 327, 5961 (Jan 2010), 81-84. 
[3] Yeates, T. O., Crowley, C. S. and Tanaka, S. Bacterial Microcompartment Organelles: Protein Shell Structure and Evolution. Annual Reviews, City, 2010.

[4] O'Neil, A., Reichhardt, C., Johnson, B., Prevelige, P. E. and Douglas, T. Genetically Programmed In Vivo Packaging of Protein Cargo and Its Controlled Release from Bacteriophage P22. Angew. Chem.-Int. Edit., 50, 32 2011), 7425-7428.

[5] Tanner, P., Onaca, O., Balasubramanian, V., Meier, W. and Palivan, C. G. Enzymatic Cascade Reactions inside Polymeric Nanocontainers: A Means to Combat Oxidative Stress. Chem.-Eur. J., 17, 16 (Apr 2011), 45524560 .

[6] van Dongen, S. F. M., Nallani, M., Cornelissen, J., Nolte, R. J. M. and van Hest, J. C. M. A Three-Enzyme Cascade Reaction through Positional Assembly of Enzymes in a Polymersome Nanoreactor. Chem.-Eur. J., 15, 5 2009), 1107-1114.

[7] Patterson, D. P., Prevelige, P. E. and Douglas, T. Nanoreactors by Programmed Enzyme Encapsulation Inside the Capsid of the Bacteriophage P22. ACS Nano, 6, 6 (Jun 2012), 5000-5009.

[8] Fiedler, J. D., Brown, S. D., Lau, J. L. and Finn, M. G. RNA-Directed Packaging of Enzymes within Virus-like Particles. Angew. Chem.-Int. Edit., 49, 50 2010), 9648-9651.

[9] Lee, L., Niu, Z. and Wang, Q. Viruses and virus-like protein assemblies - Chemically programmable nanoscale building blocks. Nano Res., 2, 5 2009), 349-364.

[10] Minten, I. J., Hendriks, L. J. A., Nolte, R. J. M. and Cornelissen, J. Controlled Encapsulation of Multiple Proteins in Virus Capsids. J. Am. Chem. Soc., 131, 49 (Dec 2009), 17771-17773.

[11] Patterson, D. P., Schwarz, B., El-Boubbou, K., van der Oost, J., Prevelige, P. E. and Douglas, T. Virus-like particle nanoreactors: programmed encapsulation of the thermostable CelB glycosidase inside the P22 capsid. Soft Matter, 8, 39 2012), 10158-10166.

[12] Worsdorfer, B., Woycechowsky, K. J. and Hilvert, D. Directed Evolution of a Protein Container. Science, 331, 6017 (Feb 2011), 589-592.

[13] Arora, P. S. and Kirshenbaum, K. Nano-tailoring: Stitching alterations on viral coats. Chemistry \& Biology, 11, 4 (Apr 2004), 418-420.

[14] For some excellent examples in which the enzymes are positioned on the exterior of viruses readers are referred to the work of Evans et al, N., 4, 5640-5645 and Liu et al, ACS Nano (2012), 6, 8692-8701.

[15] Aljabali, A. A., Barclay, J. E., Steinmetz, N. F., Lomonossoff, G. P. and Evans, D. J. Controlled immobilisation of active enzymes on the cowpea mosaic virus capsid. Nanoscale, 4, 18 (Sep 21 2012), 5640-5645. 
[16] Destito, G., Schneemann, A. and Manchester, M. Biomedical Nanotechnology Using Virus-Based Nanoparticles. City, 2009.

[17] Manchester, M. and Singh, P. Virus-based nanoparticles (VNPs): Platform technologies for diagnostic imaging. Advanced Drug Delivery Reviews, 58, 14 (Dec 1 2006), 1505-1522.

[18] Steinmetz, N. F., Lin, T., Lomonossoff, G. P. and Johnson, J. E. Structure-Based Engineering of an Icosahedral Virus for Nanomedicine and Nanotechnology. City, 2009.

[19] Bruckman, M. A., Soto, C. M., McDowell, H., Liu, J. L., Ratna, B. R., Korpany, K. V., Zahr, O. K. and Blum, A. S. Role of Hexahistidine in Directed Nanoassemblies of Tobacco Mosaic Virus Coat Protein. ACS Nano, 5, 3 (Mar 2011), 1606-1616.

[20] Gerasopoulos, K., McCarthy, M., Banerjee, P., Fan, X., Culver, J. N. and Ghodssi, R. Biofabrication methods for the patterned assembly and synthesis of viral nanotemplates. Nanotechnology, 21, 5 (2010-Feb-5 2010), 055304-055304.

[21] Liu, N., Wang, C., Zhang, W., Luo, Z., Tian, D., Zhai, N., Zhang, H., $\mathrm{Li}$, Z., Jiang, X., Tang, G. and $\mathrm{Hu}, \mathrm{Q}$. Au nanocrystals grown on a betterdefined one-dimensional tobacco mosaic virus coated protein template genetically modified by a hexahistidine tag. Nanotechnology, 23, 33 (2012Aug-24 2012), 335602-335602.

[22] Wen, A. M., Lee, K. L., Yildiz, I., Bruckman, M. A., Shukla, S. and Steinmetz, N. F. Viral nanoparticles for in vivo tumor imaging. Journal of visualized experiments : JoVE, 69 (2012 Nov 2012), e4352-e4352.

[23] Soto, C. M. and Ratna, B. R. Virus hybrids as nanomaterials for biotechnology. Curr Opin Biotechnol, 21, 4 (Aug 2010), 426-438.

[24] Carette, N., Engelkamp, H., Akpa, E., Pierre, S. J., Cameron, N. R., Christianen, P. C. M., Maan, J. C., Thies, J. C., Weberskirch, R., Rowan, A. E., Nolte, R. J. M., Michon, T. and Van Hest, J. C. M. A virus-based biocatalyst. Nat. Nanotechnol., 2, 4 (Apr 2007), 226-229.

[25] Comellas-Aragones, M., Engelkamp, H., Claessen, V. I., Sommerdijk, N. A., Rowan, A. E., Christianen, P. C., Maan, J. C., Verduin, B. J., Cornelissen, J. J. and Nolte, R. J. A virus-based single-enzyme nanoreactor. Nat Nanotechnol, 2, 10 (Oct 2007), 635-639.

[26] de la Escosura, A., Nolte, R. J. M. and Cornelissen, J. J. L. M. Viruses and protein cages as nanocontainers and nanoreactors. Journal of Materials Chemistry, 19, 16 2009), 2274-2278.

[27] Dunker, A. K. and Fernandez, A. Engineering productive enzyme confinement. Trends Biotechnol., 25, 5 (May 2007), 189-190. 
[28] Glasgow, J. E., Capehart, S. L., Francis, M. B. and Tullman-Ercek, D. Osmolyte-Mediated Encapsulation of Proteins inside MS2 Viral Capsids. ACS Nano, 6, 10 (Oct 23 2012), 8658-8664.

[29] Auton, M., Roesgen, J., Sinev, M., Holthauzen, L. M. F. and Bolen, D. W. Osmolyte effects on protein stability and solubility: A balancing act between backbone and side-chains. Biophysical Chemistry, 159, 1 (Nov 2011), 90-99.

[30] Khan, S. H., Ahmad, N., Ahmad, F. and Kumar, R. Naturally Occurring Organic Osmolytes: From Cell Physiology to Disease Prevention. Iubmb Life, 62, 12 (Dec 2010), 891-895.

[31] Minten, I. J., Nolte, R. J. M. and Cornelissen, J. J. L. M. Complex Assembly Behavior During the Encapsulation of Green Fluorescent Protein Analogs in Virus Derived Protein Capsules. Macromolecular Bioscience, 10, 5 (May 14 2010), 539-545.

[32] Inoue, T., Kawano, M. A., Takahashi, R. U., Tsukarnoto, H., Enornoto, T., Imai, T., Kataoka, K. and Handa, H. Engineering of SV40based nano-capsules for delivery of heterologous proteins as fusions with the minor capsid proteins VP2/3. J. Biotechnol., 134, 1-2 (Mar 2008), 181-192.

[33] Ellis, R. J. Macromolecular crowding: obvious but underappreciated. Trends Biochem.Sci., 26, 10 (Oct 2001), 597-604.

[34] Zhou, H. X., Rivas, G. N. and Minton, A. P. Macromolecular crowding and confinement: Biochemical, biophysical, and potential physiological consequences. Annual Reviews, City, 2008.

[35] Minten, I. J., Claessen, V. I., Blank, K., Rowan, A. E., Nolte, R. J. M. and Cornelissen, J. J. L. M. Catalytic capsids: the art of confinement. Chem Sci, 2, 2 2011), 358-362.

[36] Witus, L. S. and Francis, M. B. Using Synthetically Modified Proteins to Make New Materials. Accounts Chem. Res., 44, 9 (Sep 2011), 774-783.

[37] Ellis, R. J. Macromolecular crowding: an important but neglected aspect of the intracellular environment. Curr. Opin. Struct. Biol., 11, 1 (Feb 2001), 114-119.

[38] Minton, A. P. The influence of macromolecular crowding and macromolecular confinement on biochemical reactions in physiological media. J. Biol. Chem., 276, 14 (Apr 2001), 10577-10580. 